\title{
Evaluation of Different Machine Learning Models and Novel Deep Learning-based Algorithm for Landslide Susceptibility Mapping
}

\section{Tingyu Zhang}

key laboratory of degraded and unused land consoliation engineering

Huanyuan Wang ( $\square$ whysxdj2021@163.com)

Key laboratory of degreded and unused land consolidation engineering

Tianqing Chen

Key Laboratory of degraded and unused land consoliation engineering

\section{Zenghui Sun}

Key laboratory of degraded and unused land consoliation engineering

\section{Tao Wang}

Shaanxi Provincial Land Engineering Construction Group

\section{Dan Luo}

Shaanxi Provincial Land Engineering Construction Group

\section{Chao Li}

Shaanxi Provincial Land Engineering Construction Group

\section{Yanan Li}

key laboratory of degraded and unused land consolidation engineering

\section{Ling Han}

Changan University: Chang'an University

\section{Research Letter}

Keywords: Landslide susceptibility, Deep learning, Kernel logistic regression, Support vector machine, Evaluation

Posted Date: July 28th, 2021

DOl: https://doi.org/10.21203/rs.3.rs-720898/v1

License: (c) (1) This work is licensed under a Creative Commons Attribution 4.0 International License. Read Full License 
1 Evaluation of different machine learning models and novel

2 deep learning-based algorithm for landslide susceptibility

3 mapping

4 Tingyu Zhang a,b, Huanyuan Wang a,b, , Tianqing Chen ${ }^{\text {a,b }}{ }^{\text {a, }}$ Zenghui Sun ${ }^{\text {a,b }}$, Tao

5 Wang ${ }^{c}$, Dan Luo ${ }^{c}$, Chao Li ${ }^{\text {c }}$, Yanan Li ${ }^{\text {a,b }}$, Ling Han d

$6{ }^{a}$ Key Laboratory of Degraded and Unused Land Consolidation Engineering, the

7 Ministry of Natural Resources, Xi'an, Shaanxi, China.

$8 \quad{ }^{b}$ Institute of Land Engineering and Technology, Shaanxi Provincial Land Engineering

9 Construction Group Co., Ltd., Xi'an, Shaanxi, China.

${ }^{c}$ Shaanxi Provincial Land Engineering Construction Group Land Survey Planning and Design Institute Co., Ltd., Xi'an, Shaanxi, China.

${ }^{d}$ School of Land Engineering, Chang'an University, Xi'an, Shaanxi, China.

* Corresponding author at: Key Laboratory of Degraded and Unused Land Consolidation Engineering, the Ministry of Natural Resources, Institute of Land Engineering and Technology, Shaanxi Provincial Land Engineering Construction Group Co., Ltd., Xi'an 710064, Shaanxi, China.

Email address: whysxdj2021@163.com (Huanyuan Wang). 


\section{Abstract}

The losses and damage caused by landslides are countless in the world every year. However, the existing approaches of landslide susceptibility mapping cannot fully meet the requirement of landslide prevention, and further excavation and innovation are also needed. Therefore, the main aim of this study is to develop a novel deep learning model namely landslide net (LSNet) to assess the landslide susceptibility in Hanyin County, China, meanwhile, support vector machine model (SVM) and kernel logistic regression model (KLR) were employed as reference model. The inventory map was generated based on 259 landslides, the training dataset and validation dataset were respectively prepared using $70 \%$ landslides and the remaining 30\% landslides. The variance inflation factor (VIF) was applied to optimize each landslide predisposing factor. Three benchmark indices were used to evaluate the result of susceptibility mapping and area under receiver operating characteristics curve (AUROC) was used to compare the models. Result demonstrated that although the processing speed of LSNet model is the slowest, it still significantly outperformed its corresponding benchmark models with validation dataset, and has the highest accuracy (0.950), precision (0.951), F1 (0.951) and AUROC (0.941), which reflected excellent predictive ability in some degree. The achievements obtained in this study can improve the rapid response capability of landslide prevention for Hanyin County.

Keywords: Landslide susceptibility; Deep learning; Kernel logistic regression; Support vector machine; Evaluation 


\section{Instruction}

Landslide is defined as the special geological phenomenon that is threatening to mankind triggering by human activities or natural factors. Under the dual background of human activities and natural transmutations, the occurrence rate of landslides in the world increased rapidly(Sun et al., 2020). In addition, the landslides seriously threaten the safety of human life and property. In the face of increasingly serious landslide threats, the development of disaster prevention and mitigation work can effectively reduce the threat posed by landslides. In order to plan and construct the city safely and effectively, and to carry out the work of disaster prevention and mitigation successfully, it is necessary to quantitatively assess the landslide susceptibility on the regional scale. The first step of regional landslide susceptibility assessment (LSA) is to collect the development characteristics and spatial distribution features of historical and hidden danger landslides(Pradhan and Lee, 2010). Then the predisposing factors of landslide occurrence are selected from the geological and environment background. Subsequently, the linear or non-linear mapping relationship between predisposing factors and the degree of landslide susceptibility is analyzed by using qualitative or quantitative method, and the contribution rate of each landslide predisposing factor is determined. In the end, some techniques of analysis and comparison are used to choose the suitable model for the study area(Carrara et al., 1995).

With the development of geographic information system (GIS) and satellite remote sensing technology within each subject area, GIS-based statistical method was introduced in the field of LSA. On the whole, these basic statistical methods of LSA 
can be summarized into two categories: linear regression analysis and non-linear regression analysis. For example, certainty index model(Fan et al., 2017), statistical index model(Razavizadeh et al., 2017), logistic regression model(Aditian et al., 2018; Pourghasemi et al., 2013) and probability theory method belong to the linear regression analysis method. Neural network model(Polykretis and Chalkias, 2018; SOMA et al., 2019), support vector machine model (SVM)(Bui et al., 2016; Pandey and Pourghasemi, 2020), limit learning model and composite exponential model belong to the non-linear regression analysis method. Although researchers have done a lot of studies using these basic statistical methods, the results of LSA are not all satisfactory(Bui et al., 2018). Due to data quality, factor selection, model parameter adjustment and other factors, some low accuracy, over fitting, and owe fitting problems often appear. In order to solve these problems, hybrid model was developed in recent years, such as reduced error pruning trees (REPT)(Pham et al., 2019b), kernel logistic regression model integrated

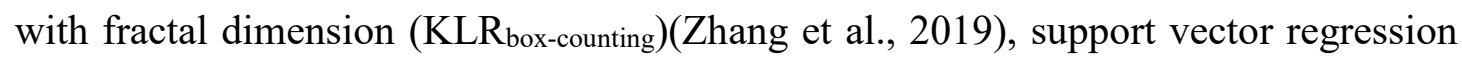
model integrated with gray wolf optimization algorithm (SVR-GWO)(Balogun et al., 2021), adaptive neuro-fuzzy inference system model integrated with satin bowerbird optimizer algorithms (ANFIS-SBO)(Chen et al., 2021). Although several models listed above have been previously applied in assessment field of landslide susceptibility and performed well, applying these models to forecast landslide occurrence and explore how to raise prediction accuracy are still the focus of current researches.

Recently, deep learning (DL) technique, a part of machine learning, is gradually applied in various fields. For example, Panahi (2020) used convolutional neural 
networks and recurrent neural networks to predict the probability of flash flood(Panahi et al., 2020); Kumar (2020) used deep learning model to complete the prediction of ground water depth(Kumar et al., 2020); Benzekri (2020) employed the deep learning model to construct an early forest fire detection system(Benzekri et al., 2020). In general, DL model performed a satisfactory ability of classification and regression. The main reason is that DL is completely a data-driven feature learning method, and has multi-level non-linear operations, which can abstractly represent classification features from a large amount of data, and combines gradient transfer method to optimize its endto-end network structure(Zhu et al., 2020). However, it is seldom used in the study of LSA.

Therefore, this study proposed a novel deep learning network named LSNet that composed of multiple convolutional layer to predict the landslide susceptibility in Hanyin County, Shaanxi Province, China. The patches of landslide predisposing factor maps were used as the input data to train the LSNet, meanwhile the LSI was regarded as the output to predict the landslide susceptibility. In addition, the support vector machine model (SVM) and kernel logistic regression model (KLR) were employed to compare with LSNet. The primary difference here between this study and the literature mentioned is that approaches existed in this paper are seldom used and compared in landslide susceptibility assessment, especially LSNet and KLR. Another point is that three models were first applied in Hanyin County and the proposed deep learning network aims to improve the accuracy of LSA in the study area. Finally, all the results may help the government to make efficient decisions about landslide prevention and 
111

112

provide prevention references for landslide risk.

\section{Sample description of study area}

Hanyin County belongs to the hilly area in southern Shaanxi Province, the geographical coordinates are $32^{\circ} 68^{\prime}-33^{\circ} 09^{\prime}$ north latitude and $108^{\circ} 11^{\prime}-108^{\circ} 44^{\prime}$ east longitude (Figure 1). The study area is about $51 \mathrm{Km}$ wide from east to west, $58 \mathrm{Km}$ long from north to south, and covers an area of about $1347 \mathrm{Km}^{2}$. The climate type of study area is continental tropical monsoon climate and the temperature varies greatly. According to the local meteorological statistics, the mean annual precipitation in the past 50 years is about $920 \mathrm{~mm}$, and the rainfall in the northern region is significantly less than that in the southern region. The water resources in the study area are very abundant, and there are 4 rivers in total, all of which belong to Yangtze River system. There are three types of groundwater in the study area, including loose rock pore water, carbonate fissure water, and bedrock fissure water.

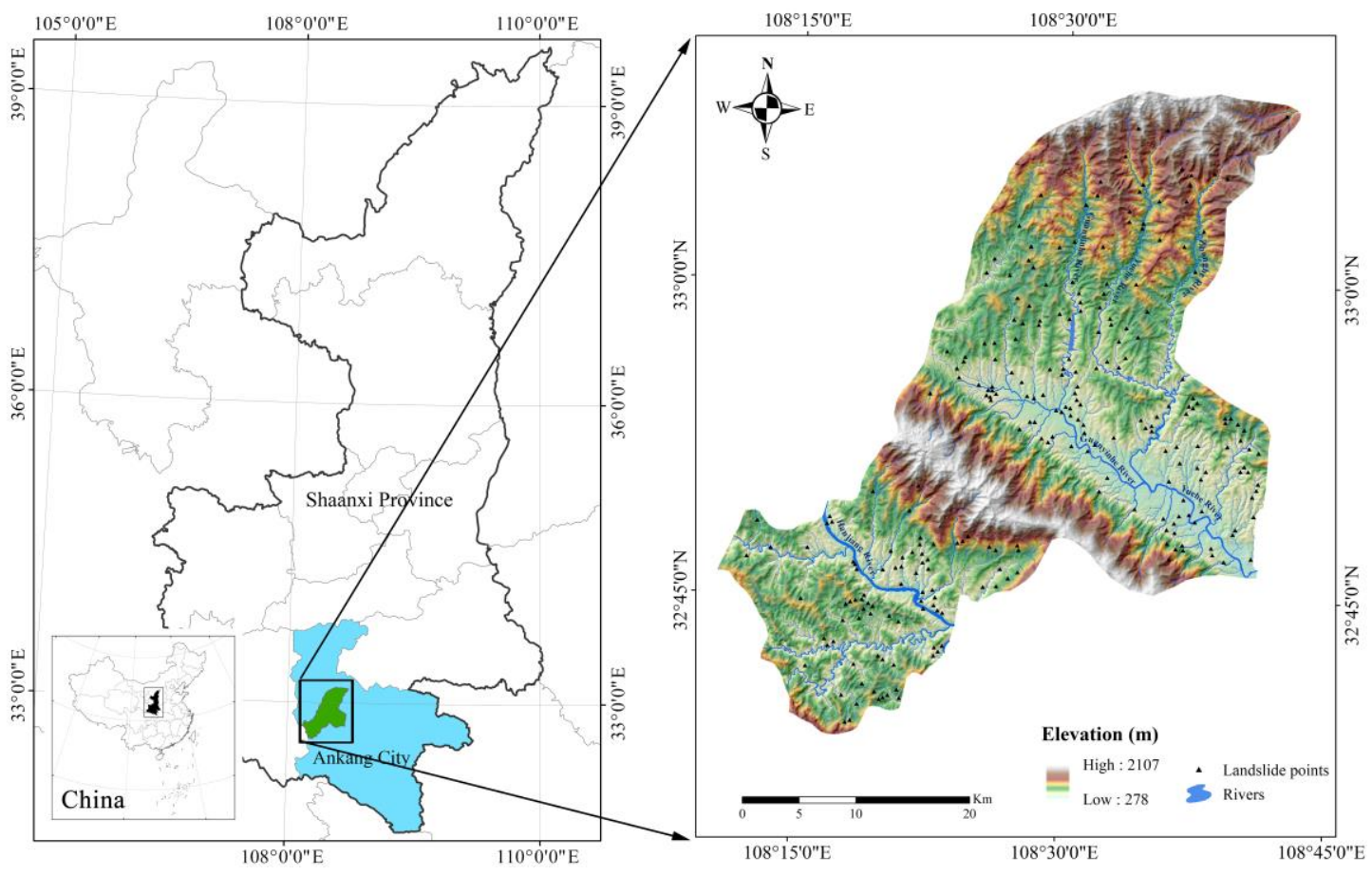

Fig. 1 The location and landslide inventory map of study area 
127 valleys, hills and basins, and the area of mountains accounts for $87 \%$. The exposed

128 strata and main lithology in the study area are shown in the Table 1. Since the

129 geotectonic location of the study area is located in the core zone of the Qinling

130 microplate, there are many faults and folds in this area. In fact, there are a total of 5

131 faults that have been proven. Besides, according to the historical records, there have

132 been 16 earthquakes in the study area, with an average magnitude of 4 , but these

133 earthquakes did not cause major damage.

Table 1 The main lithology information of the study area

\begin{tabular}{lll}
\hline Geological Age & Symbol & Main lithology \\
\hline Quaternary & Q & Sandy clay, Clay rock \\
Tertiary & E & Clay rock, Siltstone, Glutenite \\
Middle Devonian & $\mathrm{D}_{2}$ & Limestone, Calcium schist \\
Lower Devonian & $\mathrm{D}_{1}$ & Calcium schist, Calcium sandstone, Granite \\
Silurian & $\mathrm{S}$ & Phyllite and siliceous roc, Sandstone \\
Ordovician & & \\
Cambrian & $\mathrm{O}$ & Argillaceous limestone, Carbonaceous schist, Quartzite \\
Senian & $\mathrm{Z}$ & Limestone, Slate, Phyllite \\
\hline & &
\end{tabular}

\section{Data preparation}

3.1 Landslide inventory

137 Before carrying out the LSA, it is critical to verify about the information of landslides

138 in the study area. Landslide inventory is to integration of landslide boundaries, locations, 
139

types and so on, which is the subsequent basis of data analysis and model construction. Based on the historical landslide data(PRC, 2020; SBGMR, 1989), remote sensing image(Cloud, 2020), literatures(Liu and Huang, 2006) and field survey, a total of 267 landslide were identified and mapped to generate the landslide inventory map of study area (Figure 1).

\subsection{Data preparation}

In order to prepare the input dataset for model construction, 267 landslide samples were separated into two parts according to the ratio of 7/3(Zhao and Chen, 2020). Among them, 187 landslide samples were used as the training dataset to train the model, and the remaining 80 landslide samples were applied as the validation dataset to finish the validation purpose.

\subsection{Analysis and quantification of landslide predisposing factors}

In this study, we purposed altitude, slope angle, slope aspect, normalized difference vegetation index (NDVI), distance to rivers, distance to roads, distance to faults, mean annual precipitation (MAP) and lithology as the landslide predisposing factors. Since the original attribute data of each predisposing factor is very different, the frequency ratio (FR) is introduced to unify the dimension of each predisposing factor. The calculation process of FR value is shown in the Equation (1).

$$
\mathrm{FR}=\mathrm{Sam}_{\mathrm{ij}} / \operatorname{Are}_{\mathrm{ij}}
$$

Where $\mathrm{Sam}_{\mathrm{ij}}$ stands for the percentage of landslides in each landslide predisposing factor class, and $\mathrm{Are}_{\mathrm{ij}}$ is the area percentage of each landslide predisposing factor class(Siahkamari et al., 2017). 

predisposing factors, and the data sources, resolution and classification result of each predisposing factor map are listed in Table 2.

Table 2 The information of landslide predisposing factors

\begin{tabular}{|c|c|c|c|}
\hline Landslide predisposing factors & Original format & Resolution & Classification method \\
\hline Altitude (m) & grid & $30 \mathrm{~m} \times 30 \mathrm{~m}$ & natural break (Jenks) \\
\hline Slope angle $\left({ }^{\circ}\right)$ & grid & $30 \mathrm{~m} \times 30 \mathrm{~m}$ & natural break (Jenks) \\
\hline Slope aspect & grid & $30 \mathrm{~m} \times 30 \mathrm{~m}$ & natural break (Jenks) \\
\hline NDVI & grid & $30 \mathrm{~m} \times 30 \mathrm{~m}$ & natural break (Jenks) \\
\hline Distance to rivers (m) & vector & $30 \mathrm{~m} \times 30 \mathrm{~m}$ & Equal interval \\
\hline Distance to roads (m) & vector & $30 \mathrm{~m} \times 30 \mathrm{~m}$ & Equal interval \\
\hline Distance to faults (m) & vector & $30 \mathrm{~m} \times 30 \mathrm{~m}$ & Equal interval \\
\hline MAP (mm/year) & vector & $30 \mathrm{~m} \times 30 \mathrm{~m}$ & Equal interval \\
\hline Lithology & vector & $30 \mathrm{~m} \times 30 \mathrm{~m}$ & Custom interval \\
\hline
\end{tabular}

\section{Methodologies}

The main research contents include 4 parts: (1) Using the data that already available to

167 complete the landslide inventory; (2) Using FR value to quantify the landslide predisposing factor maps, and partitioning dataset; (3) Using the factor maps that

169 already quantified by FR to train the SVM model and KLR model, moreover using the original factor maps to train the LSNet model; (4) Producing LSM corresponding to

171 each model, assessing the result accuracy, and comparing the prediction performance

172 of each model. The flowchart of this study is shown in the Figure 2. The techniques 
used in this study is described as follows.

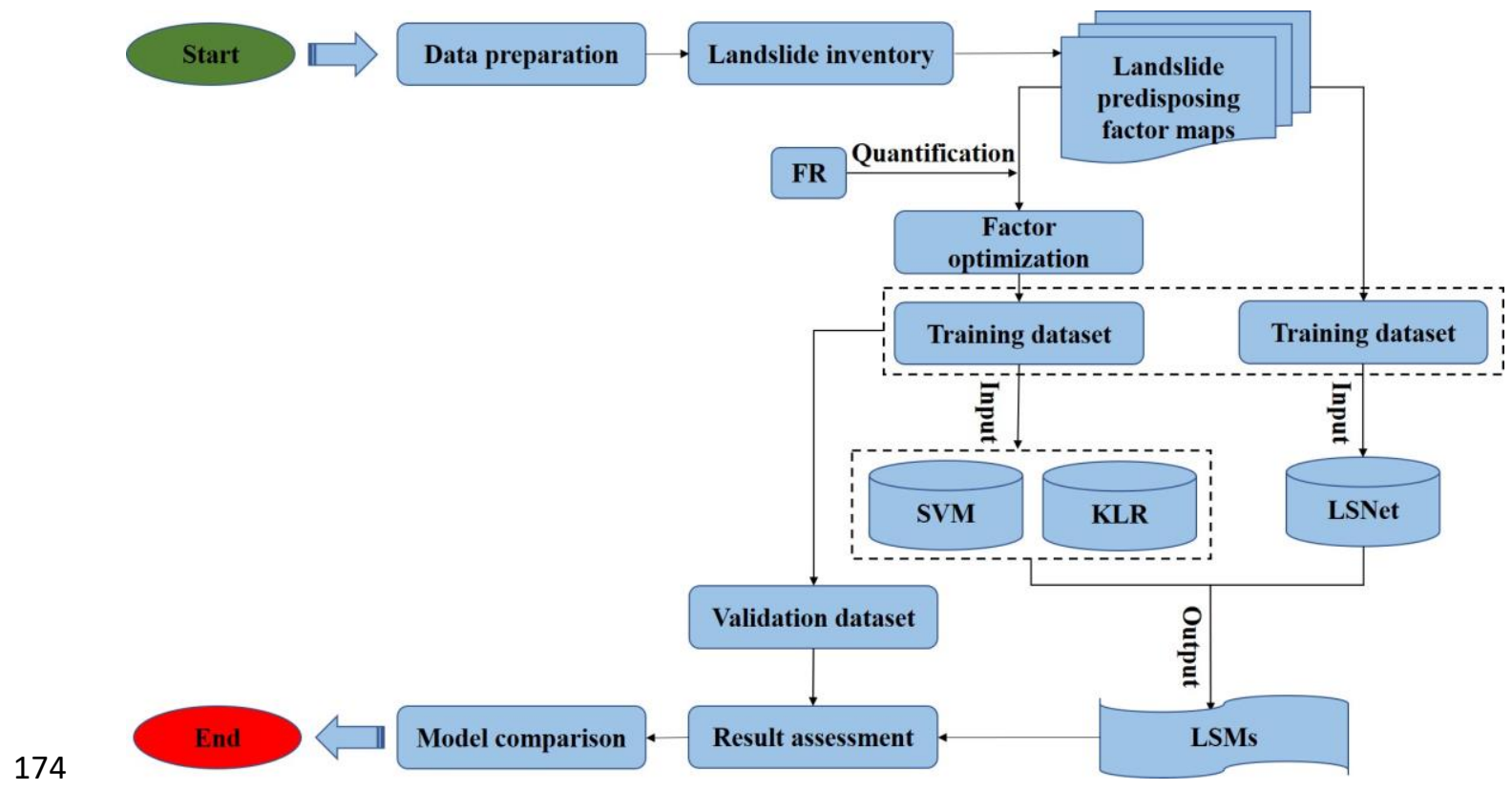

Fig. 2 The flowchart of the study

176

177

\subsection{Factor optimization method}

Since the assumption of machine learning modeling is that the variables are independent of each other, it needs to detect whether there is strong correlation between the factors. This strong correlation relationship is called multicollinearity which may cause the over fitting or under fitting problems(Hong et al., 2018). In this study, the variance inflation factor (VIF) and tolerances (TOL) were applied to reflect the multicollinearity problem, which can be calculated by constructing a linear regression model based on the training dataset. When $\mathrm{VIF}>10$ and $\mathrm{TOL}<0.1$, it indicates that the predisposing factor has a multicollinearity problem and needs to be eliminated, vice versa(Pham et al., 2019a).

4.2 Support vector machine model (SVM)

The basic principle of SVM is to search the optimal separating hyperplane that can maximize the interval between positive and negative samples in training dataset(Wang 
and Brenning, 2021). Initially, SVM model was used as the supervised learning algorithm to solve binary classification problem, while the non-linear classification problem can be solved after introducing the kernel function. Therefore, the SVM model was applied in many researches about landside susceptibility assessment. In addition, there are three parameters namely penalty factor $\left(C_{0}\right)$, non-sensitive loss function $(\varepsilon)$, and kernel function parameter $(\gamma)$ that need to be adjusted appropriately in the process of constructing the SVM model(Xie et al., 2021). The main steps of SVM model construction can be described as below.

At first, the landslide predisposing factors are defined as the dataset of instance label pairs $\left(s_{i}, t_{i}, \mathrm{i}=1,2, \ldots, \mathrm{n}\right)$, where $s_{i}$ stands for the input data, $t_{i}$ is the output classes (landslide and non-landslide), and $\mathrm{n}$ is the number of training samples(Kumar et al., 2017). The training samples are mapped in to a $n$-dimensional hyperplane by using the RBF kernel function which can be defined as:

$$
K\left(s_{i}, s_{j}\right)=\left(-\gamma\left(s_{i}-s_{j}\right)\right), \quad \gamma>0
$$

Then mathematical expression of the n-dimensional hyperplane $L$ needs to satisfy the following condition:

$$
t_{j}\left(w \cdot s_{j}+b\right)+\varepsilon \geq 1
$$

Where $w$ denotes for the norm of normal hyperplane, and $b$ is the constant. The maximum interval between vector and hyperplane can be derived by applying the Lagrangian multiplier(Abedini et al., 2019), and cost function can be expressed as:

$$
L=1 / 2\|w\|^{2}-C_{0} \sum_{i=1}^{n} \varepsilon
$$


In statistical learning, when there are phenomena such as non-linear estimation, nonnormal estimation, and uneven variance, it may cause invalid estimation by using the ordinary regression method(Chen et al., 2018). These problems were overcome after the introduction of logistic regression, and logistic regression is widely used to solve binary classification problem. However, the structure of original logistic regression model is relatively simple, the flexibility is relatively low, and it still has defects in dealing with non-linear classification problems(Chen et al., 2019). While the kernel function can help to solve these problems effectively in constructing logistic regression model. Therefore, the hybrid model namely kernel logistic regression is created. In order to be consistent with the SVM model above, the RBF kernel function is determined to build KLR model. The expression of KLR model is as follows:

$$
p_{i}\left(t=1 \mid k_{i}\right)=\frac{1}{1+e^{-\left(k_{i}+\alpha\right)}}
$$

Where $p_{i}$ is the probability of landslide occurrence, $k_{i}$ stands for the $i$ th row of $K\left(s_{i}\right.$, $s_{j}$ ), and $\alpha$ is a constant for the intercept(Thai and Indra, 2018).

\subsection{Landslide net model (LSNet)}

The deep learning has been widely used in the field of remote sensing image processing, including change detection, land use classification, image registration and so on. The deep belief networks, convolutional neural network (CNN), and auto coder are the three most commonly used network models in deep learning. The operating principle of these networks is to stack multiple layers within the model, and use the output of the previous item as the input of the next item, so that the features of each layer in the network can be converted into higher-dimensional features(Bui et al., 2020). Among them, the CNN 


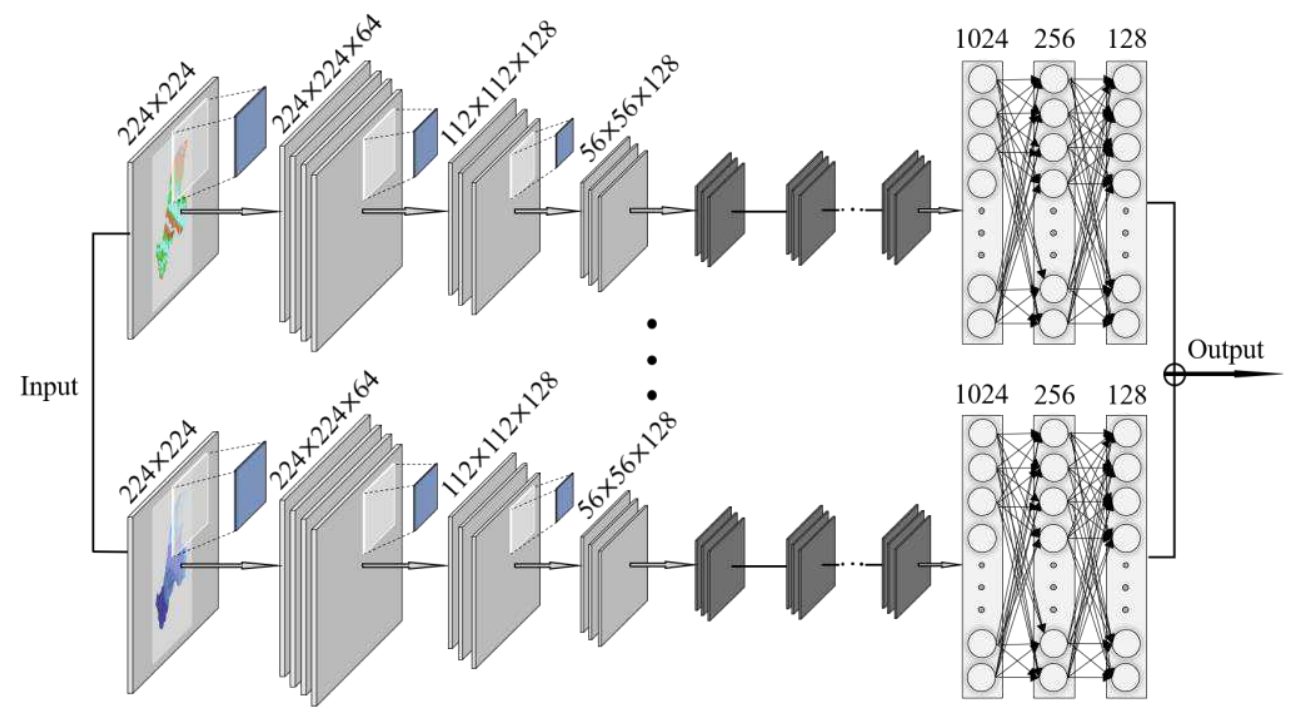

Fig. 3 The structure schematic diagram of landslide net (LSNet)

LSNet is a multi-layer feedforward neural network, the advantage of which is that it can process large-scale data in the form of multiple arrays from the local and global input data. The structure of LSNet is consist of multiple layers, which are related to each other through a set of learnable weights and biases. The local and global scale features can be captured by these convolutional blocks using scanning of the entire image. Meanwhile, the pooling layer and rectified linear unit (ReLU) layer are used for generalization to improve the non-linear fitting ability of the network(Li et al., 2021). Additionally, each convolutional layer contains feature maps obtained by multiple convolution kernels, and these feature maps share the node weights of the convolution kernels, so features can be extracted from different parts. Specifically, the main operation performing in $\mathrm{CNN}$ can be generalized as follows:

$$
O^{l}=\operatorname{pool}_{p}\left(\sigma\left(O^{l-1} * W^{l}+b^{l}\right)\right)
$$



represent the weight and deviation of input feature layer convoluting by linear

251 convolution, and $\sigma$ is a non-linear function outside the convolution layer.

2524.5 Assessment and comparison method

\subsubsection{Result assessment method}

In order to assess the accuracy of classification result and compare the performance of

\begin{tabular}{lll}
\hline Samples & Landslide & Non-landslide \\
\hline Landslide & True positive (TP) & True negative (TN) \\
Non-landslide & False positive (FP) & False negative (FN) \\
\hline
\end{tabular}
each model, statistical indexes are purposed to finish this work. A matrix (Table 3 ) is constructed by true positive (TP), false positive (FP), true negative (TN), and false negative (FN) calculating from training dataset(Pham et al., 2021). The accuracy and precision are calculated according to the Equation (7) and (8) for accuracy assessment, meanwhile, the consistency of the results is verified with F1. The calculation process is as follows.

Table 3 Discriminant matrix of statistical indexes 
indicators including the running speed of the model, the classification ability for landslide and non-landslide, and the generalized performance of the model. Among them, based on the validation dataset, the running speed of the model is quantitative expressed by time, and the sensitivity and specificity are respectively used to reflect the classification ability for landslide and non-landslide (Equation (9) and (10))(Yanar et al., 2020). Additionally, the receiver operating characteristics curve (ROC) is used for assessing the generalized performance, and in general, the larger the area under ROC curve (AUROC), the stronger the generalization ability of the model(Dang et al., 2020).

$$
\begin{aligned}
& \text { Sensitivtiy }=\frac{T P}{T P+F N} \\
& \text { Specificity }=\frac{T N}{T N+F P}
\end{aligned}
$$

\section{Results}

5.1 The quantification results of FR for landslide predisposing factors

In this study, the FR value was employed to quantify each landslide predisposing factor according to the classification result. It can be observed from the Table 4 that the interval of Tertiary from the lithology factor has the highest $F R$ value $(F R=2.32)$, followed by the range of $<100$ from the distance to roads factor $(\mathrm{FR}=1.85)$, and the range of 278-548 from the altitude factor $(F R=1.81)$. On the contrary, the lowest FR value appears in both the 1432-2107 interval of the altitude factor $(F R=0.00)$ and the flat interval of the slope factor $(F R=0.00)$.

Table 4 The FR calculation result for each class of landslide predisposing factors

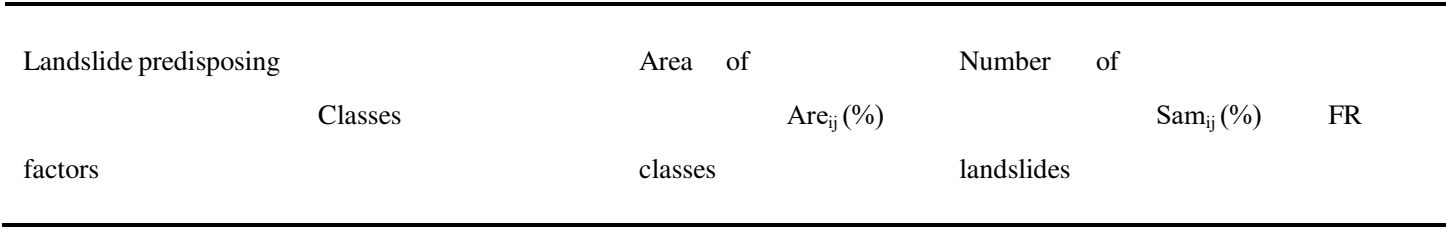




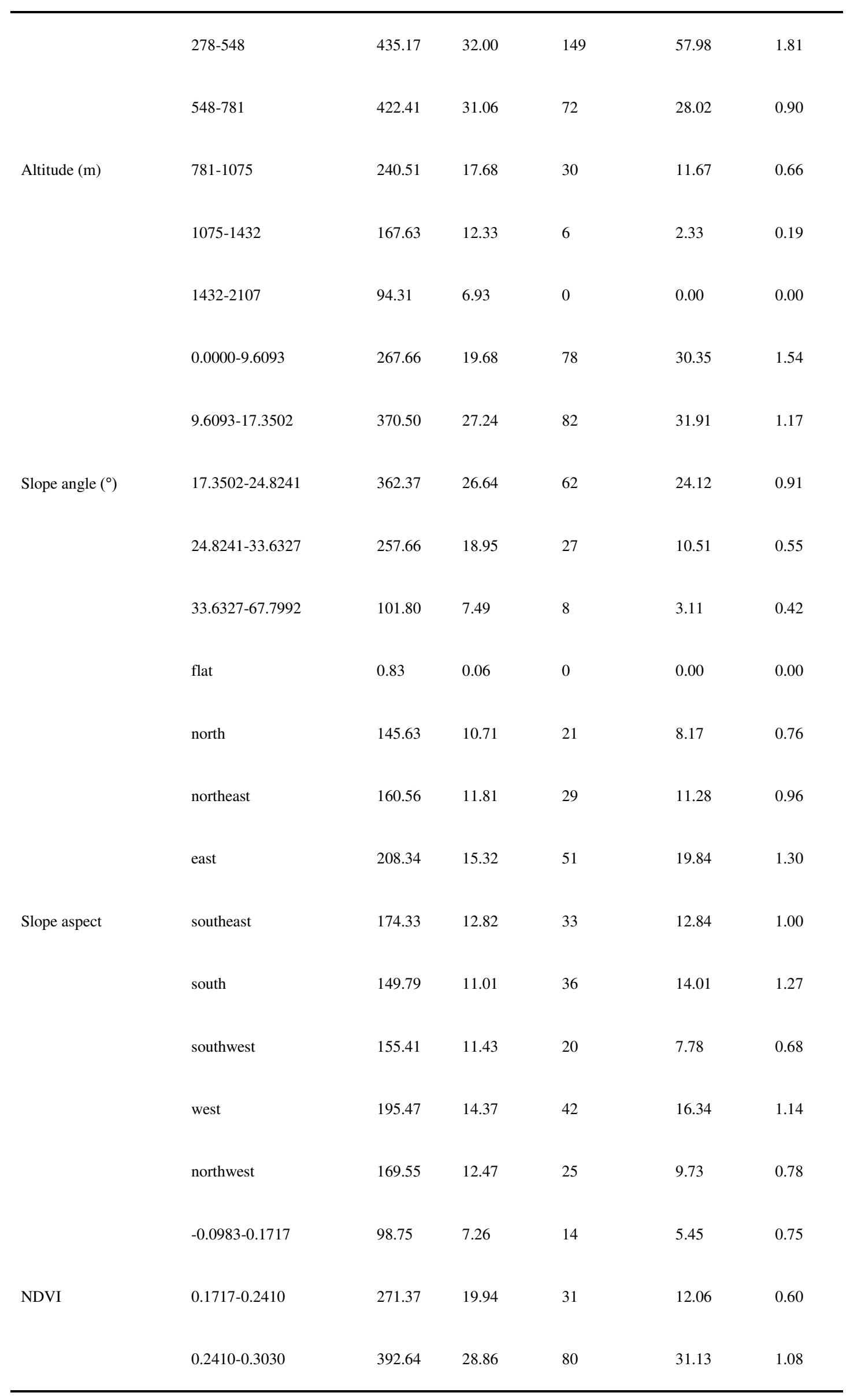




\begin{tabular}{|c|c|c|c|c|c|c|}
\hline & $0.3030-0.3698$ & 392.02 & 28.81 & 90 & 35.02 & 1.22 \\
\hline & $0.3698-0.5308$ & 205.88 & 15.13 & 42 & 16.34 & 1.08 \\
\hline \multirow{5}{*}{ Distance to rivers (m) } & $<100$ & 97.84 & 7.19 & 22 & 8.56 & 1.19 \\
\hline & $100-200$ & 74.62 & 5.48 & 16 & 6.23 & 1.14 \\
\hline & $200-300$ & 70.25 & 5.16 & 19 & 7.39 & 1.43 \\
\hline & $300-400$ & 67.24 & 4.94 & 20 & 7.78 & 1.57 \\
\hline & $>400$ & 1050.86 & 77.22 & 180 & 70.04 & 0.91 \\
\hline \multirow{5}{*}{ Distance to roads (m) } & $<100$ & 85.67 & 6.30 & 30 & 11.67 & 1.85 \\
\hline & $100-200$ & 73.42 & 5.39 & 21 & 8.17 & 1.51 \\
\hline & $200-300$ & 65.54 & 4.82 & 20 & 7.78 & 1.62 \\
\hline & $300-400$ & 61.18 & 4.50 & 16 & 6.23 & 1.38 \\
\hline & $>400$ & 1075.02 & 79.00 & 170 & 66.15 & 0.84 \\
\hline \multirow{5}{*}{ Distance to faults (m) } & $<1000$ & 157.90 & 11.60 & 50 & 19.46 & 1.68 \\
\hline & $1000-2000$ & 147.95 & 10.87 & 33 & 12.84 & 1.18 \\
\hline & $2000-3000$ & 132.95 & 9.77 & 30 & 11.67 & 1.19 \\
\hline & $3000-4000$ & 120.84 & 8.88 & 25 & 9.73 & 1.10 \\
\hline & $>4000$ & 801.18 & 58.87 & 119 & 46.30 & 0.79 \\
\hline \multirow{5}{*}{ Lithology } & Quaternary & 246.08 & 18.02 & 73 & 28.40 & 1.58 \\
\hline & Tertiary & 50.43 & 3.69 & 22 & 8.56 & 2.32 \\
\hline & Middle Devonian & 129.17 & 9.46 & 5 & 1.95 & 0.21 \\
\hline & Lower Devonian & 32.82 & 2.40 & 5 & 1.95 & 0.81 \\
\hline & Silurian & 61.36 & 4.49 & 5 & 1.95 & 0.43 \\
\hline
\end{tabular}




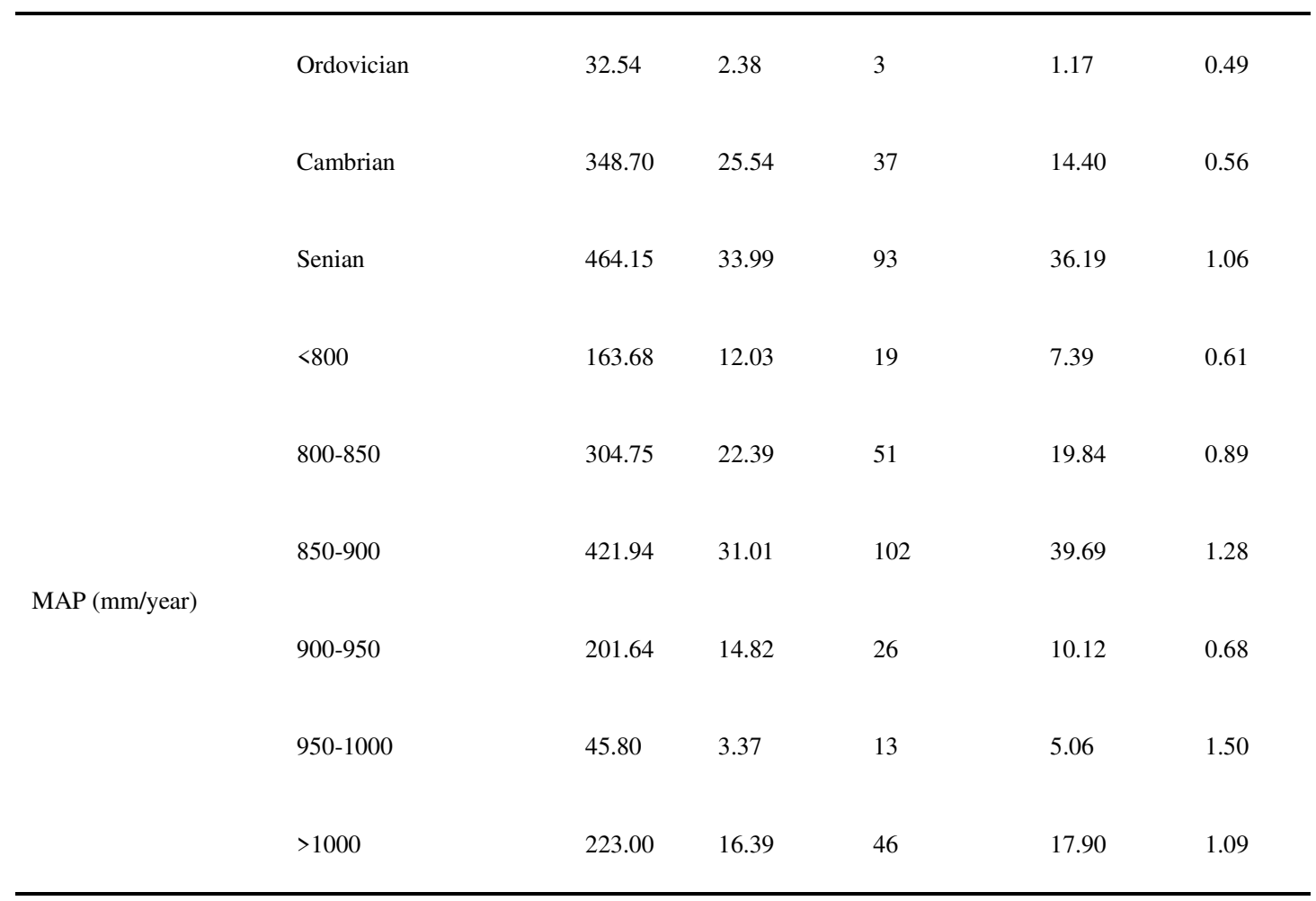

288

5.2 The optimization result of landslide predisposing factors

The VIF and TOL values of each landslide predisposing factor were calculated based on the quantified landslide predisposing factors, and the calculation results were shown in the Table 5. As can be seen from the results, the largest VIF value and the smallest TOL value appear in NDVI (VIF=1.433, TOL $=0.698)$, followed by the altitude $(\mathrm{VIF}=1.293, \mathrm{TOL}=0.773)$ and the aspect $(\mathrm{VIF}=1.268, \mathrm{TOL}=0.789)$. By contrast, the distance to roads has the smallest VIF value and the largest TOL value (VIF=1.019, TOL $=0.981)$. Since the VIF and TOL values of all landslide predisposing factors are not inside the critical range $(\mathrm{VIF}>10$ and $\mathrm{TOL}<0.1)$, all factors are retained and used to prepare the dataset.

Table 5 The VIF and TOL values of each landslide predisposing factor

\begin{tabular}{lll}
\hline Landslide predisposing factors & VIF & Tolerances (TOL) \\
\hline Altitude & 1.293 & 0.773
\end{tabular}




$\begin{array}{llc}\text { Slope angle } & 1.032 & 0.969 \\ \text { Aspect } & 1.268 & 0.789 \\ \text { MAP } & 1.044 & 0.958 \\ \text { Lithology } & 1.103 & 0.907 \\ & & 0.871 \\ \text { Distance to rivers } & 1.148 & 0.928 \\ & & \\ \text { Distance to faults } & 1.078 & 0.981 \\ \text { Distance to roads } & 1.019 & 0.698\end{array}$
datasets were prepared according to aforementioned partition principle. Subsequently,

301 the training dataset was used as the input data to implement the following three models.

3025.3 Implementation of SVM model

In this study, the training dataset was used to construct the SVM model. Since the parameters of RBF kernel function are significant for model construction, the 10 -fold 305 cross validation method was used to search the most suitable parameter set $\left(C_{0}, \gamma\right)$. The 306 optimized parameter set is $(241,0.02)$. Then run the trained SVM model in the python 307 platform, and adjust the output range of the model to $0.000-1.000$ which also represents 308 the LSI. In the end, the natural break (Jenks) method was used to divide the LSI into 309 five ranges which respectively represent the very low susceptibility area $(0.0899$ 310 0.2084), low susceptibility area (0.2085-0.4646), moderate susceptibility area (0.4647311 0.6228), high susceptibility area (0.6229-0.7893) and very high susceptibility area 312 (0.7894-0.9224), furthermore the LSM was generated by converting these areas to 


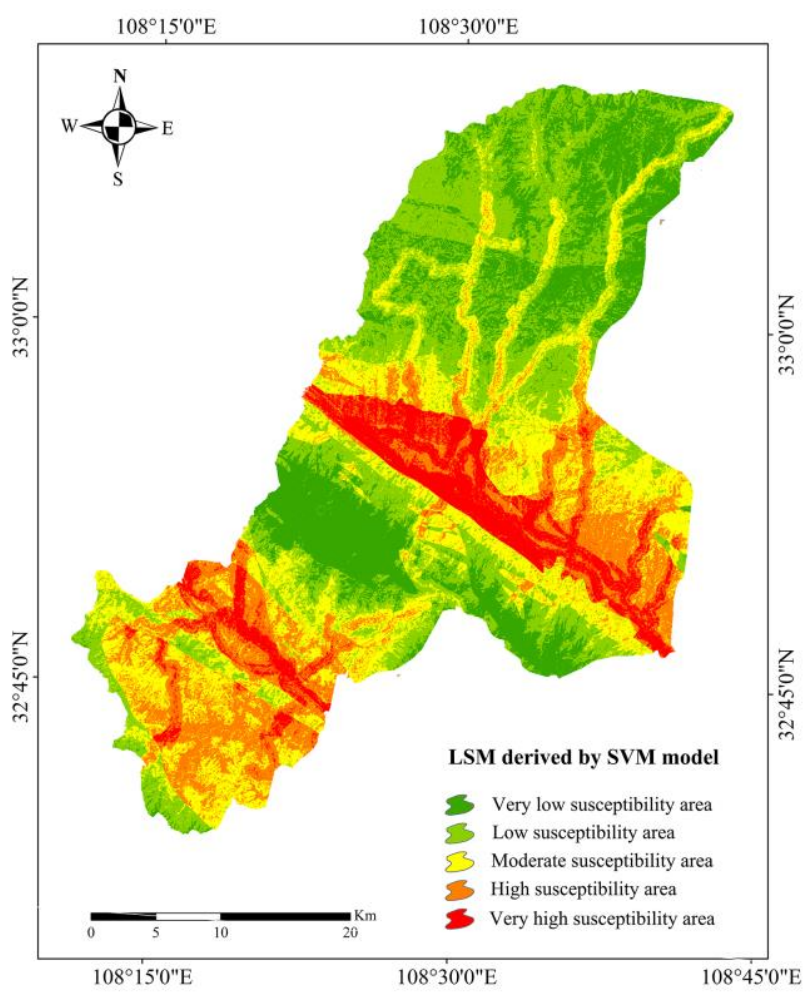

Fig. 4 Landslide susceptibility map of study area derived by SVM model

5.4 Implementation of KLR model

The construction progress of KLR model is similar with the SVM model. For the purpose of comparison, the parameter set $\left(C_{0}, \gamma\right)$ was consistent with that of the SVM model. Subsequently, the training dataset was used as the input data for KLR model construction in the python platform, and adjust the output range of the LSI to 0.0001.000. Finally, the LSI was divided into five ranges by using the natural break (Jenks) method. These five ranges respectively represent the very low susceptibility area

$323(0.0145-0.2459)$, low susceptibility area $(0.2460-0.3695)$, moderate susceptibility area $324(0.3696-0.5161)$, high susceptibility area (0.5162-0.6974) and very high susceptibility 325 area (0.6975-0.9983), moreover the LSM corresponding to KLR model was generated in ArcGIS software (Figure 5). 


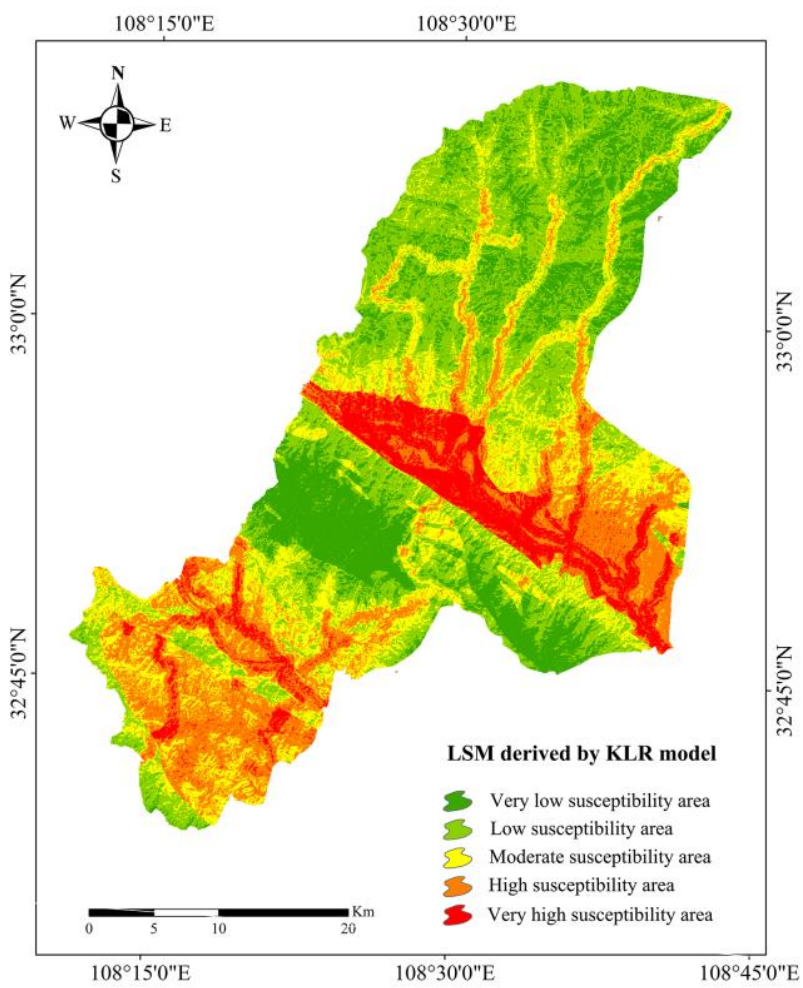

Fig. 5 Landslide susceptibility map of study area derived by KLR model

The LSNet was coded using tensorflow 2.0 under the python environment, and running on a personal computer with Intel(R) Core(TM) i7-7700k CPU, RTX 3080Ti GPU, 32 GB RAM, and the Windows 10 operating system. The LSNet had multi-layer structure, the size of input window was designed as $224 \times 224$. AlexNet can implement more than 1000 categories of classification, in contrast, the landslide susceptibility mapping is a binary classification problem, which does not require deep network design. For this reason, in this study, the size of input layer in convolution kernel of LSNet was set to $5 \times 5$, the size of the convolution kernel for the other layers was set to $3 \times 3$, the number of feature maps for each layer was set to $64,128,128,256,256$, respectively. At the same time, a pooling layer, non-linear activation function ReLU and batch normalized 

epoch are set as 0.0001 and 600 to learn the depth features through back propagation.

Subsequently, the number of neurons in the fully connected layers was set to 1024,256 , occurrence to output confidence, namely LSI.

(Figure 6).

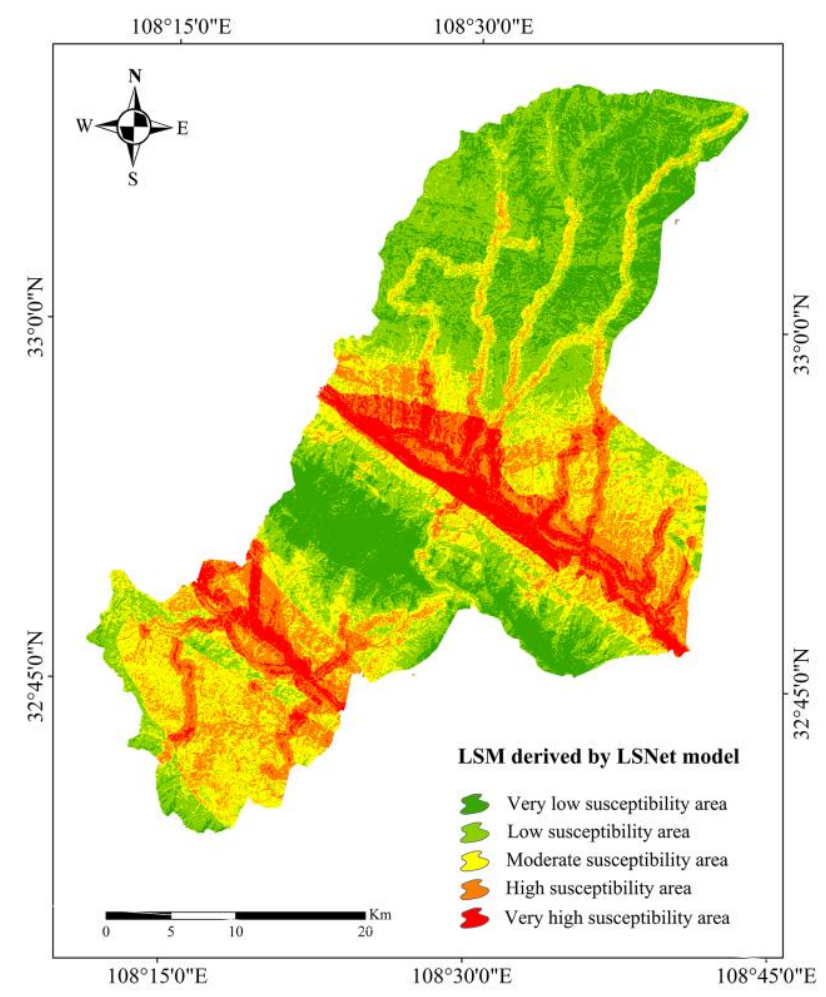

Fig. 6 Landslide susceptibility map of study area derived by LSNet model 
355 After mapping the LSMs of these three models, it is necessary to assess the quality of 356 results. In this study, the matrix has been organized based on the validation dataset, then 357 the accuracy, precision, and F1 values for each LSM were calculated (Table 6). As 358 shown in Table 6, the LSNet model gets the highest accuracy value and precision value 359 (accuracy $=0.950$, precision $=0.951$ ), by contrast, the SVM model gets the lowest 360 accuracy value and precision value (accuracy $=0.825$, precision $=0.850$ ), while the 361 performance of the KLR model is moderate. From the value of F1, the LSNet also gets 362 the highest value $(\mathrm{F} 1=0.951)$, followed by the KLR model and SVM model, which is 363 also consistent with the ordering of accuracy and precision values.

364 Table 6 Calculation results of statistical indexes for landslide susceptibility mapping

\begin{tabular}{llll}
\hline Parameters & SVM & KLR & LSNet \\
\hline TP & 34 & 36 & 39 \\
TN & 32 & 36 & 37 \\
FP & 6 & 6 & 2 \\
FN & 8 & 2 & 2 \\
Accuracy & & & \\
Precision & 0.825 & 0.900 & 0.950 \\
F1 & 0.850 & 0.857 & 0.951 \\
Sensitivity & & & 0.951 \\
& 0.829 & 0.900 & 0.951 \\
\hline
\end{tabular}

366 In order to compare the running speed, classification and generalization performance, 

generalization ability.

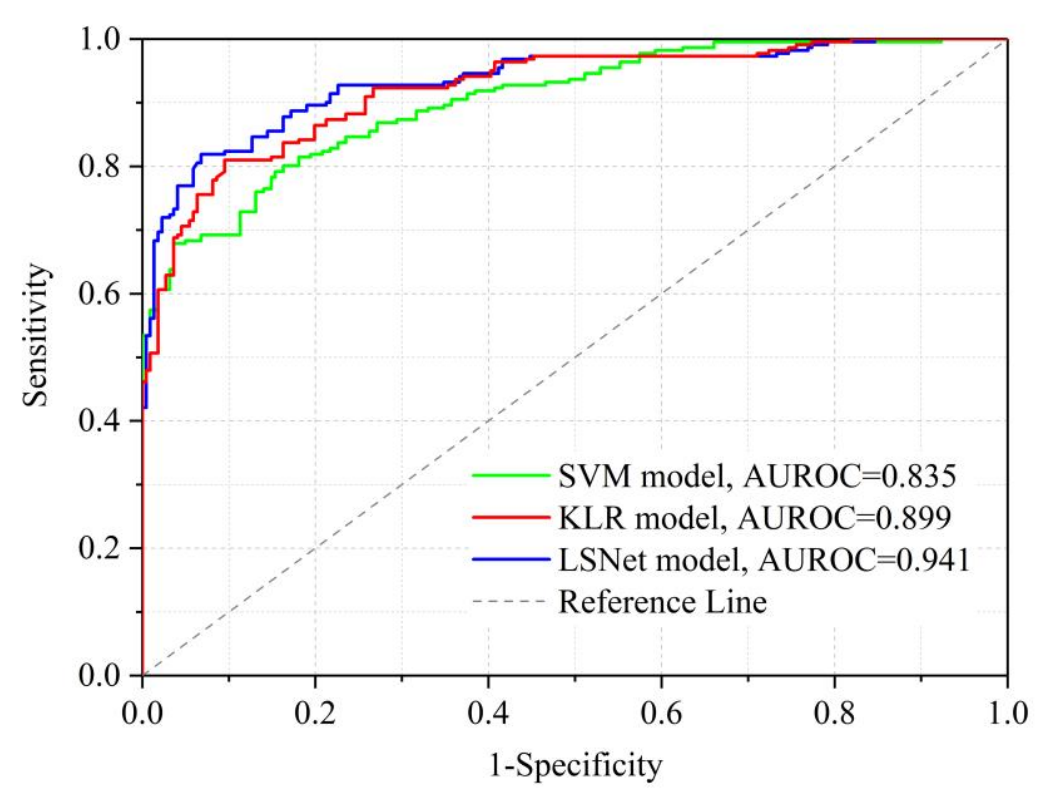
For AUROC values (Figure 7), the LSNet model also obtains the largest AUROC value (AUROC=0.941), followed by the KLR model (AUROC=0.899) and SVM model (AUROC $=0.835$ ), and the results show that the LSNet model has the best

Fig. 7 The ROC curves of each landslide susceptibility model based on validation dataset 
while the running speed of the LSNet model (107s) is significantly slower than the first models.

\section{Discussion}

In this paper, we show the progress and results of landslide susceptibility mapping based on SVM model, KLR model, and LSNet model in Hanyin County, Shaanxi Province, China. In terms of the model performance, although the classification accuracy of the three models is higher, the accuracy of LSNet and other statistical indexes are higher than that of SVM and KLR, which fully shows that the LSNet performs best in the study area.

Since both SVM and KLR are developed based on statistical theory, the quality of input data and the adjustment of model parameters in the process of model construction may affect the final result. Before preparation of input datasets, three classification methods i.e. natural break (Jenks), equal interval, and custom interval were all used to grade FR-quantified landslide predisposing factors. However, the classification methods and results of landslide predisposing factors are inevitably affected by human factors, which may lead to over-fitting or under-fitting(Yacine and Pourghasemi, 2019). For this reason, it is necessary to deeply analyze the impact of classification methods on data quality. Besides, this study only used two machine learning models for comparison, therefore, more models should be added for reference in subsequent research, so that the advantages and disadvantages of deep learning and machine learning in landslide susceptibility mapping can be more comprehensively compared. In contrast, as a deep learning model, the input data of LSNet is a complete remote 
sensing image containing all the information. In order to distinguish landslide and nonlandslide from image data, not only the objects in the image patch need to be characterized as landslides, but also need to accurately and reliably represent the contextual information of the landslide space background. The advantage of LSNet is to derive the category of the object at the image block level, and learn the spatial distribution through the CNN network with hierarchical representation, and finally obtain the probability of each object's category through multiple fully connected layers and softmax. It is different from machine learning in principle, and its specific advantages include: (1) LSNet can classify based on object blocks in a deep learning network of convolutional structure, and output the category probability; (2) LSNet uses the $\mathrm{CNN}$ model to learn the internal and overall spatial information of the object block to represent the contextual spatial semantic information of the category; LSNet represents the probability of the category at the object block level, which can avoid pixel-level misfits and improve the accuracy of classification(Dimililer et al., 2021). Interestingly, the running time of LSNet is significantly longer than that of SVM and KLR, which may be limited by the hardware performance of the computer, resulting in slower calculations. Nevertheless, this does not mean that the LSNet is not a state of art model and other studies have reached similar conclusions in their researches.

On the other hand, as a black box model, DL cannot intuitively reflect the spatial distribution features of landslides in the study area during data preparation. On the contrary, in machine learning modeling, because FR is used to quantify the graded landslide predisposing factors, the spatial distribution of the landslide under the 
conditions of each predisposing factor can be intuitively reflected from the quantified results(Zhang et al., 2020). For instance, from the view of distance to rivers and roads, as the distance from roads and rivers increase, the FR value decreases, indicating that the closer to the river and the road, the more landslides are distributed. This is because the exposed rock and soil in study area have low mechanical strength, the surface is easily weathered and eroded, and the joints and fissures are very developed. Moreover, due to the scouring action from the river and excavation of the slope toe during road construction, the original stress structure of the slope was destroyed, which resulted in the instability of the slope and generated a large number of potential landslides. This consistent with the phenomenon we observed in the field, and is similar to the results of geological hazard studies in similar areas of the study area(Liu et al., 2020; Wang et al., 2016).

\section{Conclusion}

Landslide susceptibility mapping is a key step for landslide prevention work. This study used Hanyin County, Shaanxi Province, China as the study area to finish the work of landslide susceptibility mapping by building the LSNet model, SVM model, and KLR model, and generated the LSM. Then various of statistical indexes was applied for the accuracy assessment, and the ROC curves was employed to compare the performance and classification ability of the models. In summary, the main conclusions are as follows: (1) In the process of dataset preparation and parameter adjustment, the machine learning model will inevitably be affected by human factors, resulting in unstable classification results. However, LSNet can overcome human interference and generate 
objective classification results. (2) LSNet can avoid the problems of over-fitting and under-fitting. The classification accuracy in the study area is high, moreover the generalization is stronger than the SVM model and the KLR model. The LSNet can be promoted and used in the study area.

In addition, this study introduced the construction method of LSNet model in detail, and compared the performance of LSNet model (deep learning), SVM model (machine learning), and KLR model (hybrid model), which can provide reference for the application of deep learning model in landslide prevention in the future. Furthermore, the results of this study can improve the efficiency of landslide prevention for government decision-making in similar study areas, which is conducive to rapid response of landslide warning.

Author Contributions: Conceptualization, Tingyu Zhang and Huanyuan Wang; Methodology, Tianqing Chen; Software, Dan Luo; Validation, Tingyu Zhang; Formal Analysis, Zenghui Sun; Investigation, Ling Han; Resources, Chao Li; Data Curation, Tao Wang; Writing-Original Draft Preparation, Tingyu Zhang; Writing-Review \& Editing, Tingyu Zhang and Huanyuan Wang; Visualization, Yanan Li; Supervision, Ling Han; Project Administration, Tingyu Zhang; Funding Acquisition, Tingyu Zhang. All authors have read and agreed to the published version of the manuscript.

Funding: This study is financially supported by Fundamental Research Funds for the Central Universities (300102351502), Opening Fund of Key Laboratory of Land Remediation of Shaanxi Province, and Inner scientific research project of Shaanxi Land Engineering Construction Group (SXDJ2021-10) (SXDJ2021-30) (SXDJ2020-22). 
471 Conflicts of Interest: The authors declare no conflict of interest.

472 References

473 Abedini, M., Ghasemian, B., Shirzadi, A., Bui, D.T., 2019. A comparative study of support vector machine and logistic model tree classifiers for shallow landslide susceptibility modeling. Environmental Earth Sciences, 78, 560-577.

Aditian, A., Kubotab, T., Shinoharab, Y., 2018. Comparison of GIS-based landslide susceptibility models 
494

495

Carrara, A., Cardinali, M., Guzzetti, F., Reichenbach, P., 1995. Gis Technology in Mapping Landslide Hazard. Geographical Information Systems in Assessing Natural Hazards, 8, 135-175.

Chen, W., Chen, X., Peng, J., Panahi, M., Lee, S., 2021. Landslide susceptibility modeling based on ANFIS with teaching-learning-based optimization and Satin bowerbird optimizer. Geoscience Frontiers, 12, 93-107.

Chen, W., Shahabi, H., Shirzadi, A., Hong, H., 2018. Novel hybrid artificial intelligence approach of bivariate statistical-methods-based kernel logistic regression classifier for landslide susceptibility modeling. Bulletin of Engineering Geology and the Environment, 78, 4397-4419.

Chen, W. et al., 2019. Spatial prediction of landslide susceptibility using data mining-based kernel logistic regression, naive Bayes and RBFNetwork models for the Long County area (China). Bulletin of engineering geology and the environment, 78, 247-266.

Cloud, G.D., 2020. GDEMV2 30M resolution digital elevation data, GDEMV2 30M resolution digital elevation data. Geospatial Data Cloud, http://www.gscloud.cn/sources/accessdata/421?pid=302.

Dang, V.-H., Hoang, N.-D., Nguyen, L.-M.-D., Samui, P., Samui, P., 2020. A Novel GIS-Based Random Forest Machine algorithm for Spatial Prediction of Shallow Landslide Susceptibility. Forests, 113-129.

Dimililer, K., Dindar, H., Al-Turjman, F., 2021. Deep learning, machine learning and internet of things in geophysical engineering applications: An overview. Microprocessors and Microsystems, 80, 103-613.

Fan, W., Wei, X.S., Cao, Y.B., Zheng, B., 2017. Landslide susceptibility assessment using the certainty factor and analytic hierarchy process. Journal of Mountain Science, 21, 100-119. 
Hong, H. et al., 2018. Landslide susceptibility mapping using J48 Decision Tree with AdaBoost, Bagging and Rotation Forest ensembles in the Guangchang area (China). Catena, 163, 399-413.

Kumar, D., Roshni, T., Singh, A., Jha, M.K., Samui, P., 2020. Predicting groundwater depth fluctuations using deep learning, extreme learning machine and Gaussian process: a comparative study. Earth Science Informatics, 13, 1-14.

Kumar, D., Thakur, M., S.Dubey, C., P.Shukla, D., 2017. Landslide susceptibility mapping \& prediction using Support Vector Machine for Mandakini River Basin, Garhwal Himalaya, India. Geomorphology, 295, 115-125.

Li, W., Fang, Z., Wang, Y., 2021. Stacking ensemble of deep learning methods for landslide susceptibility mapping in the Three Gorges Reservoir area, China. Stochastic Environmental Research and Risk Assessment

Liu, H., Li, X., Meng, T., Liu, Y., 2020. Susceptibility mapping of damming landslide based on slope unit using frequency ratio model. Arabian Journal of Geosciences, 13, 178-192.

Liu, Y., Huang, Q., 2006. The formation and mechanism of an expansive soil highway landslide. Coal Geology \& Exploration, 13, 41-44.

Panahi, M. et al., 2020. Deep learning neural networks for spatially explicit prediction of flash flood probability. 12, 370-383.

Pandey, V.K., Pourghasemi, H.R., 2020. Landslide susceptibility mapping using maximum entropy and support vector machine models along the highway corridor, Garhwal Himalaya. Geocarto International, 35, 168-187.

Pham, B.T., Prakash, I., K.Singh, S., Shirzadi, A., Shahabi, H., 2019a. Landslide susceptibility modeling using Reduced Error Pruning Trees and different ensemble techniques: Hybrid machine learning 
approaches. CATENA, 175, 203-218.

Pham, B.T. et al., 2019b. Landslide susceptibility modeling using Reduced Error Pruning Trees and different ensemble techniques: Hybrid machine learning approaches. Catena, 175, 203-218.

Pham, Q.B., Yacine, A., Ali, S.A., Parvin, F., Vojtek, M., 2021. A comparison among fuzzy multi-criteria decision making, bivariate, multivariate and machine learning models in landslide susceptibility mapping. Geomatics, Natural Hazards and Risk, 12, 1741-1777.

Polykretis, C., Chalkias, C., 2018. Comparison and evaluation of landslide susceptibility maps obtained from weight of evidence, logistic regression, and artificial neural network models. Natural Hazards, 93, 249-274.

Pourghasemi, H.R., Moradi, H.R., Aghda, S.M.F., 2013. Landslide susceptibility mapping by binary logistic regression, analytical hierarchy process, and statistical index models and assessment of their performances. Natural Hazards, 69, 605 609.

Pradhan, B., Lee, S., 2010. Delineation of landslide hazard areas on Penang Island, Malaysia, by using frequency ratio, logistic regression, and artificial neural network models. Environmental Earth Sciences, 60, 1037-1054.

PRC, 2020. The Ministry of Emergency Management released the basic situation of natural disasters nationwide in 2019, http://www.gov.cn/shuju/2020-01/17/content 5470130.htm (Accessd on 17 January 2020).

Razavizadeh, S., Solaimani, K., Massironi, M., Kavian, A., 2017. Mapping landslide susceptibility with frequency ratio, statistical index, and weights of evidence models: a case study in northern Iran. Environmental Earth Sciences, 76, 499-512.

SBGMR, 1989. Regional Geology of Shaanxi Province. Geological Publishing House. (In Chinese), Bei 

Jing, China.

Siahkamari, S., Haghizadeh, A., Zeinivand, H., Tahmasebipour, N., 2017. Spatial prediction of floodsusceptible areas using frequency ratio and maximum entropy models. Geocarto International, $33,927-941$.

SOMA, A.S., KUBOTA, T., MIZUNO, H., 2019. Optimization of causative factors using logistic regression and artificial neural network models for landslide susceptibility assessment in Ujung Loe Watershed, South Sulawesi Indonesia. Journal of Mountain Science, 16, 144-162.

Sun, X. et al., 2020. Application of a GIS-based slope unit method for landslide susceptibility mapping along the rapidly uplifting section of the upper Jinsha River, South-Western China. Bulletin of Engineering Geology and the Environment, 79, 533-549.

Thai, P.B., Indra, P., 2018. Machine Learning Methods of Kernel Logistic Regression and Classification and Regression Trees for Landslide Susceptibility Assessment at Part of Himalayan Area, India. Indian Journal of Science \& Technology, 11, 1-10.

Wang, L., Guo, M., Sawada, K., Lin, J., Zhang, J., 2016. A comparative study of landslide susceptibility maps using logistic regression, frequency ratio, decision tree, weights of evidence and artificial neural network. Geosciences Journal, 20, 117-136.

Wang, Z., Brenning, A., 2021. Active-Learning Approaches for Landslide Mapping Using Support Vector Machines. Remote Sensing, 13, 2588-2607.

Xie, W. et al., 2021. Landslide hazard assessment based on Bayesian optimization-support vector machine in Nanping City, China. Natural Hazards, 26, 18-31.

Yacine, A., Pourghasemi, H.R., 2019. How do machine learning techniques help in increasing accuracy of landslide susceptibility maps? Geoscience Frontiers, 11, 328-345. 
581

582

583

584

585

586

587

588

589

590

591

592

593

594

Yanar, T., Kocaman, S., Gokceoglu, C., 2020. Use of Mamdani Fuzzy Algorithm for Multi-Hazard Susceptibility Assessment in a Developing Urban Settlement (Mamak, Ankara, Turkey). International Journal of Geo-Information, 9, 114-128.

Zhang, T. et al., 2019. Assessment of Landslide Susceptibility Using Integrated Ensemble Fractal Dimension with Kernel Logistic Regression Model. Entropy, 21, 218-234.

Zhang, Y. et al., 2020. Optimizing the frequency ratio method for landslide susceptibility assessment: a case study of the Caiyuan Basin in the southeast mountainous area of China. Journal of Mountain Science, 17, 340-357.

Zhao, X., Chen, W., 2020. Optimization of Computational Intelligence Models for Landslide Susceptibility Evaluation. Remote Sensing, 12, 2180-2200.

Zhu, L. et al., 2020. Landslide Susceptibility Prediction Modeling Based on Remote Sensing and a Novel Deep Learning Algorithm of a Cascade-Parallel Recurrent Neural Network. Sensors, 20, 15761591. 